Article

\title{
Sanitation Sustainability Index: A Pilot Approach to Develop a Community-Based Indicator for Evaluating Sustainability of Sanitation Systems
}

\author{
Shervin Hashemi ${ }^{\circledR}$ \\ Institute for Environmental Research, Yonsei University College of Medicine, 50-1 Yonsei-ro, Seodaemun-gu, \\ Seoul 03722, Korea; shervin@yuhs.ac
}

Received: 5 June 2020; Accepted: 25 August 2020; Published: 26 August 2020

\begin{abstract}
Evaluating the sustainability of sanitation systems is essential in achieving the sixth sustainable development goal. However, there are only limited number of available evaluation indexes, which are utilized to macroscopically determine a community's sanitation coverage. Consequently, an index is required, which can evaluate different sanitation options for a specific community. In this paper, the sanitation sustainability index (SSI) is suggested as an indicator for evaluating the sustainability of sanitation systems. The SSI has sub-indexes that consider the technical, social, and economic aspects of the sanitation system, and all the variables are dimensionless and heavily dependent on the current state of the community where the sanitation system is going to be implemented. The applicability of the SSI was demonstrated by evaluating the implementation of two onsite sanitation systems, including one septic tank system and one resource-oriented sanitation (ROS) system in South Korea. A sensitivity analysis defined the variables that have significant impact, and the statistical distribution of the SSI for both systems was forecasted. The results showed that for South Korea, which has a profound history of utilizing human waste as fertilizer, utilizing the resource-oriented sanitation system is more sustainable, although it has a lower social sub-index score compared to the septic tank system.
\end{abstract}

Keywords: resource-oriented sanitation; sanitation sustainability index; SDG6; sustainability assessment; sustainable development goals; sustainable sanitation

\section{Introduction}

According to UNICEF and WHO [1], the population that utilize safely managed sanitation services increased from $28 \%$ to $45 \%$ from 2000 up to 2017 . Accordingly, 2.1 billion people gained access to at least basic sanitation services. Despite that, 2 billion people still have no access to safe and sustainable sanitation system, 673 million people are practicing open defecation, and 3 billion people still lack basic sanitation; consequently, the sanitation crisis remains a global concern.

The sixth sustainable development goal (SDG6) is designed to solve these global issues before the year 2030 [2]. The objective of SDG6-2 is to provide full coverage of adequate and sustainable sanitation for the entire population. Following this objective, innovative approaches have been applied on new disciplinary understandings of sanitation, to provide sustainable sanitation for low-income regions [3]. Recently, national-scaled programs have been conducted to improve the notion of equity in sanitation to end critical challenges like open defecation [4]. Meanwhile, there are several indicators and indexes that are defined to monitor or evaluate the progress [5-7]. One example is the water, sanitation, and hygiene (WaSH) performance index suggested by Cronk et al. [8], which can be utilized to compare the performances of countries in achieving universal water, sanitation, and hygiene. The elements of this index include water and sanitation access and equity. 
The development of these indexes is essential in achieving SDG6, because they present a comparative view of the progress of each country towards SDG6. Additionally, these indexes are useful for undertaking general national-scaled decisions to further progress towards fulfilling SDG6.

In many cases, comparing two different countries based on such macroscopic indexes may be misleading, particularly when there is a notable difference between the human development indexes of those countries [9]. Nevertheless, the economic, social, and technical characteristics of each country are different. Determining an appropriate index, which is flexible, suitable for every community, and reliable for comparison of progress, is crucial. Consequently, the use of an index that is better suited to evaluate sanitation systems for the implementation in a specific community is a suitable approach, and the evaluation should be performed based on the current technical, social, and economic status of the community.

An index proposed by Lundin et al. [10], which evaluates the sustainability of sanitation systems included several technical considerations. However, waste recycling and utilization, along with social and economic indicators, were not considered in that index. Another comprehensive index was suggested by Iribarnegaray et al. [11], which assesses the sustainability of water and sanitation management systems. This index is useful, as it considers the majority of relevant issues of sustainability. However, by developing innovative sanitation systems which can recycle sanitary wastes to be utilized as resources (e.g., fertilizers), there is an emerging need for providing innovative indexes, which can consider the effect of ability for onsite waste recycling on the technical, economic, and social aspects of the community [5]. In particular, economic indicators are required to consider a broader range of issues influencing the cost and probable benefits of implementing sanitation systems in developing countries [12]. Moreover, the insufficient evaluation process for the applicability of an index through limited case studies and demonstration projects is another bottleneck on the way to finding an appropriate sustainability index.

To provide sustainable sanitation for a community, understanding the technical, economic, and social characteristics of the community is critical. Numerous technical approaches toward the provision of sustainable sanitation have been studied [13-17]. These approaches provide a range of options for sanitation, including onsite sanitation systems using water-saving toilets, septic tanks, and resource-oriented sanitation (ROS) systems [18-25]. Moreover, some studies compare different sanitation systems based on economic views $[12,26]$. Social approaches toward sustainable sanitation were also considered in multiple studies [27-31]. However, there is still a need for a reliable index that can be utilized to determine a suitable sanitation system for a community based on availability, accessibility, and sustainability.

In this paper, the sanitation sustainability index (SSI) is proposed to evaluate the sanitation systems for implementation in any community. The SSI indicates the technical, social, and economic aspects of the sanitation system. The applicability of this index is demonstrated by utilizing it to evaluate the implementation of one septic tank and one ROS system in South Korea.

\section{Materials and Methods}

\subsection{Definition of SSI}

Figure 1 presents the SSI structure, which contains technical, economic, and social sub-indexes. Each sub-index has specific dimensionless variables, having values ranging from 0 to 1 , with 0.2 intervals, indicating very low, low, medium, high, and very high criteria for evaluating the sustainability of a sanitation system.

Technical and economic sub-indexes are analyzed by simple equations, containing a fraction. The numerator of the fraction is the difference between the specific parameter describing a particular feature of the sanitation system and the current norm of the community regarding the parameter. This value is then divided by the current norm of the community regarding the parameter to indicate 
how significant this difference is. Hence, the fraction makes the sub-index dimensionless, making it useful to compare two or more systems with each other in any community.

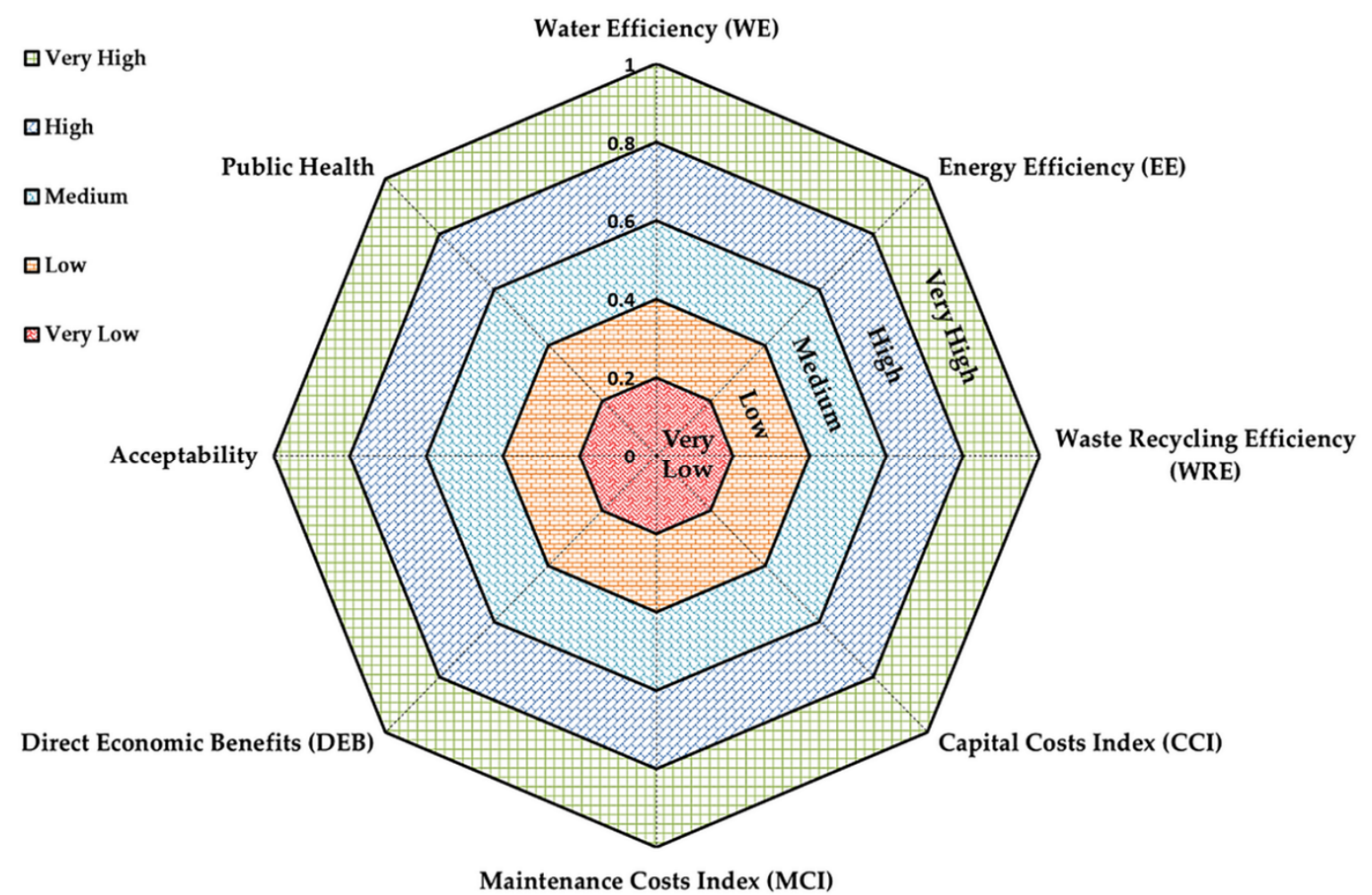

Figure 1. Structure of the sanitation sustainability index and its evaluation criteria.

Water efficiency (WE), energy efficiency (EE), and waste recycling efficiency (WRE) are dimensionless variables assigned to the technical sub-index. The WE variable is calculated by Equation (1), where $V_{C}$ is the typical amount of water consumption utilized for a single use of the sanitation system, including both water for flushing and other hygienic purposes like handwashing. $\mathrm{V}_{\mathrm{M}}$ is the mean of the volume of consumed water in the common sanitation system of the community. When $\mathrm{V}_{\mathrm{C}} \geq \mathrm{V}_{\mathrm{M}}$, the value of WE should be considered zero.

$$
\mathrm{WE}=\frac{\mathrm{V}_{\mathrm{M}}-\mathrm{V}_{\mathrm{C}}}{\mathrm{V}_{\mathrm{M}}},
$$

The EE is calculated by Equation (2), where $\mathrm{E}_{\mathrm{C}}$ is the energy consumption for treating wastewater made from a single usage of the sanitation system, and $E_{M}$ is the mean energy consumption for wastewater treatment in common sanitation systems of the community. When $E_{C} \geq E_{M}$, the value of EE should be considered zero.

$$
\mathrm{EE}=\frac{\mathrm{E}_{\mathrm{M}}-\mathrm{E}_{\mathrm{C}}}{\mathrm{E}_{\mathrm{M}}},
$$

WRE is the ratio of safely recycled waste to total waste production. This variable also evaluates the ability of a sanitation system to both treat and utilize produced waste.

For the economic sub-index, capital cost index (CCI), maintenance cost index (MCI), and direct economic benefits (DEBs) are suggested as the dimensionless variables. $\mathrm{CCI}$ and $\mathrm{MCI}$ are calculated by Equations (3) and (4), respectively. In these equations, the $C_{E}$ is the estimated capital cost, and $C C_{M}$ is the mean capital cost for the common sanitation systems of the community. Similarly, the $\mathrm{MC}_{\mathrm{E}}$ is the estimated maintenance cost for a year, and $\mathrm{MC}_{\mathrm{M}}$ is the mean maintenance cost for the common sanitation systems of the community. If $\mathrm{CC}_{\mathrm{E}} \geq \mathrm{CC}_{\mathrm{M}}$, then the value of $\mathrm{CCI}$ should be considered as zero. Similarly, if $\mathrm{MC}_{\mathrm{E}} \geq \mathrm{MC}_{\mathrm{M}}$, then the value of $\mathrm{MCI}$ should be considered as zero. 


$$
\begin{gathered}
C C I=\frac{C_{M}-C C_{E}}{C C_{M}}, \\
M C I=\frac{\mathrm{MC}_{M}-M C_{E}}{M C_{M}},
\end{gathered}
$$

DEB is a binary variable. When a sanitation system can produce economic benefits or has the potential that its economic benefit covers the capital and maintenance costs, then DEB should be 1 , otherwise, it is 0 .

The social sub-index includes acceptability and public health indicators as its relative variables. Both mentioned variables are qualitative, which must be turned into dimensionless quantitative variables through onsite investigations, and acceptability should reflect the cultural sanitation actions in the society. In this case, investigations should be performed by analyzing questionnaires that reflect the opinion of the users after experiencing the system. Therefore, the acceptability variable can be defined as the percentage of people who were satisfied after using the system and would use it again. For newly developed sanitation systems like ROS, user training can significantly increase the acceptability variable.

The public health indicator should reflect the hygiene aspects of the sanitation system. A wide range of considerations including safe treatment, recycling process, reduction of risks of incidence for sanitary diseases (e.g., diarrhea), total hygiene status of the system, especially where there is an interface with users or maintenance technicians, and efficiency in controlling public health concerns, e.g., reducing open defecation cases, are required to define this variable. Although several studies have been conducted on the social aspects of sanitation in different communities, there is no established standard survey to evaluate the acceptability and public health aspects of a sanitation system $[4,27-29,32]$. Therefore, regardless of the method of investigation, a value of 0 to 1 , representing the evaluation criteria, should be assigned for the acceptability and public health variables.

Accordingly, for both acceptability and public health, the index is flexible for any appropriate and possible local application. The scoring can be done by the decision-maker or technician who is applying this index to the results of a local investigation as well as other appropriate data.

After evaluating all the described sub-indexes and variables, the SSI score is calculated as the arithmetic mean of all the values. For a specific community, when two or more sanitation systems are being compared, the system with the higher SSI is concluded to be more sustainable for implementation, and an SSI value closer to 1 reflects higher sustainability for implementation.

\subsection{Applicability of SSI for Sanitation System Implementation in South Korea}

To demonstrate SSI applicability, the SSI is calculated to evaluate the implementation of two onsite sanitation systems: a septic tank system and an ROS system. The systems are considered for implementation in a suburban residential area of Nowon District, Seoul, Republic of Korea to offer public hygiene services. For both sanitation systems, variables related to technical and economic sub-indexes were assigned based on the characteristics and assumptions of these systems, which were described in a previous study [12]. Table 1 presents the assumptions for calculating the SSI for both sanitation systems, including references that were used for assigning specific values to each parameter. A currency exchange rate of KRW 1100 for USD 1 is used, wherever applicable.

There is insufficient research on the social acceptability and public health issues for sanitation systems in Korea. However, considering the ancient sanitation practices in Korea as described by Han \& Hashemi [5], along with other local pilot studies, it might be reasonable to consider a value of 0.7-0.8 for both social acceptability and public health indicators for the ROS system variables [20]. As using septic tank systems is quite common in both urban and rural areas of South Korea, a value of 0.9 was utilized for both the acceptability and public health variables.

A sensitivity analysis for the SSI scores calculated for both sanitation systems was conducted by applying a $\Delta= \pm 10 \%$ variation to each parameter. Additionally, to account for the lack of knowledge 
and availability of data, statistical distributions for each variable were utilized to forecast the statistical distribution of the calculated SSI indexes through Monte Carlo simulation using Oracle Crystal Ball version 11.1.2.4 with 1,000,000 trials. Details of the assumptions and results of this statistical analysis are presented in the Supplementary Material.

Table 1. Assumptions for calculating sanitation sustainability index of septic tank and resource-oriented sanitation systems in South Korea.

\begin{tabular}{|c|c|c|c|c|c|}
\hline Sub-Index & Variable & Unit & $\begin{array}{c}\text { Sanitation } \\
\text { System }\end{array}$ & $\begin{array}{c}\text { Value } \\
\text { (Range: min-max) }\end{array}$ & References \\
\hline \multirow{7}{*}{ Technical } & & & Septic Tank & $8(4-13)$ & \multirow{6}{*}[5,12,33,34]{} \\
\hline & $v_{C}$ & Liter & ROS & $1(0-2.5)$ & \\
\hline & $\mathrm{V}_{\mathrm{M}}$ & & - & 10 & \\
\hline & & \multirow{3}{*}{ Wh } & Septic Tank & $5.2(1.04-10.9)$ & \\
\hline & ${ }^{{ }^{2}} \mathrm{C}$ & & ROS & $0.65(0-2.1)$ & \\
\hline & $\mathrm{E}_{\mathrm{M}}$ & & - & 6.5 & \\
\hline & WRE & - & $\begin{array}{l}\text { Septic Tank } \\
\text { ROS }\end{array}$ & $\begin{array}{c}0.519(0.135-0.519) \\
0.9(0.7-0.9)\end{array}$ & {$[5,20,35]$} \\
\hline \multirow{5}{*}{ Economic } & $\mathrm{CC}_{\mathrm{E}}^{+}$ & \multirow{4}{*}{ US\$ } & $\begin{array}{l}\text { Septic Tank } \\
\text { ROS }\end{array}$ & $\begin{array}{l}3380.00(3121.00-9436.00) \\
4668.18(2899.22-5002.77)\end{array}$ & \multirow{5}{*}{ [12] } \\
\hline & $\mathrm{CC}_{\mathrm{M}}^{+}$ & & - & 6061.00 & \\
\hline & $\mathrm{MC}_{\mathrm{E}}^{+}$ & & $\begin{array}{l}\text { Septic Tank } \\
\text { ROS }\end{array}$ & $\begin{array}{l}2240.16(2118.18-3401.82) \\
2195.28(1687.58-2702.92)\end{array}$ & \\
\hline & $\mathrm{MC}_{\mathrm{M}}^{+}$ & & - & 2931.82 & \\
\hline & DEB & - & $\begin{array}{l}\text { Septic Tank } \\
\text { ROS }\end{array}$ & $\begin{array}{l}0 \\
1\end{array}$ & \\
\hline \multirow{2}{*}{ Social } & Acceptability & - & $\begin{array}{l}\text { Septic Tank } \\
\text { ROS }\end{array}$ & $\begin{array}{c}0.9 \\
0.75\end{array}$ & \multirow{2}{*}[16,27,28]{} \\
\hline & Public Health & - & $\begin{array}{l}\text { Septic Tank } \\
\text { ROS }\end{array}$ & $\begin{array}{c}0.9 \\
0.75\end{array}$ & \\
\hline
\end{tabular}

${ }^{\dagger}$ Including laboring costs for initialization and one-year maintenance services.

\section{Results}

\subsection{SSI Applicability for Evaluating the Proposed Implementation of Sanitation Systems in South Korea}

Figure 2 presents the results of the SSI sub-indexes evaluation for both mentioned sanitation systems in South Korea. The SSI was quantified as 0.42 and 0.71 for septic tank and ROS systems, respectively. Thus, notable differences between the calculated SSIs were observed, particularly for the ROS system, which shows better sustainability in the technical and economic sub-indexes. As described in previous studies, the ability of ROS systems to recycle waste and their economic benefits is significant [12,20]. According to the Republic of Korea, Ministry of Environment, there is an average recycling rate of $51.9 \%$ for wastewater generated from wastewater treatment facilities, such as septic tanks, in South Korea [35]. The wastewater produced in septic tank sanitation systems includes a mixture of urine, feces, hygienic materials (e.g., toilet paper), and flushing water. However, properly designed ROS systems can have a significantly higher WRE, approximately 0.7-0.9, by storing and treating urine and feces separately $[5,20]$. 


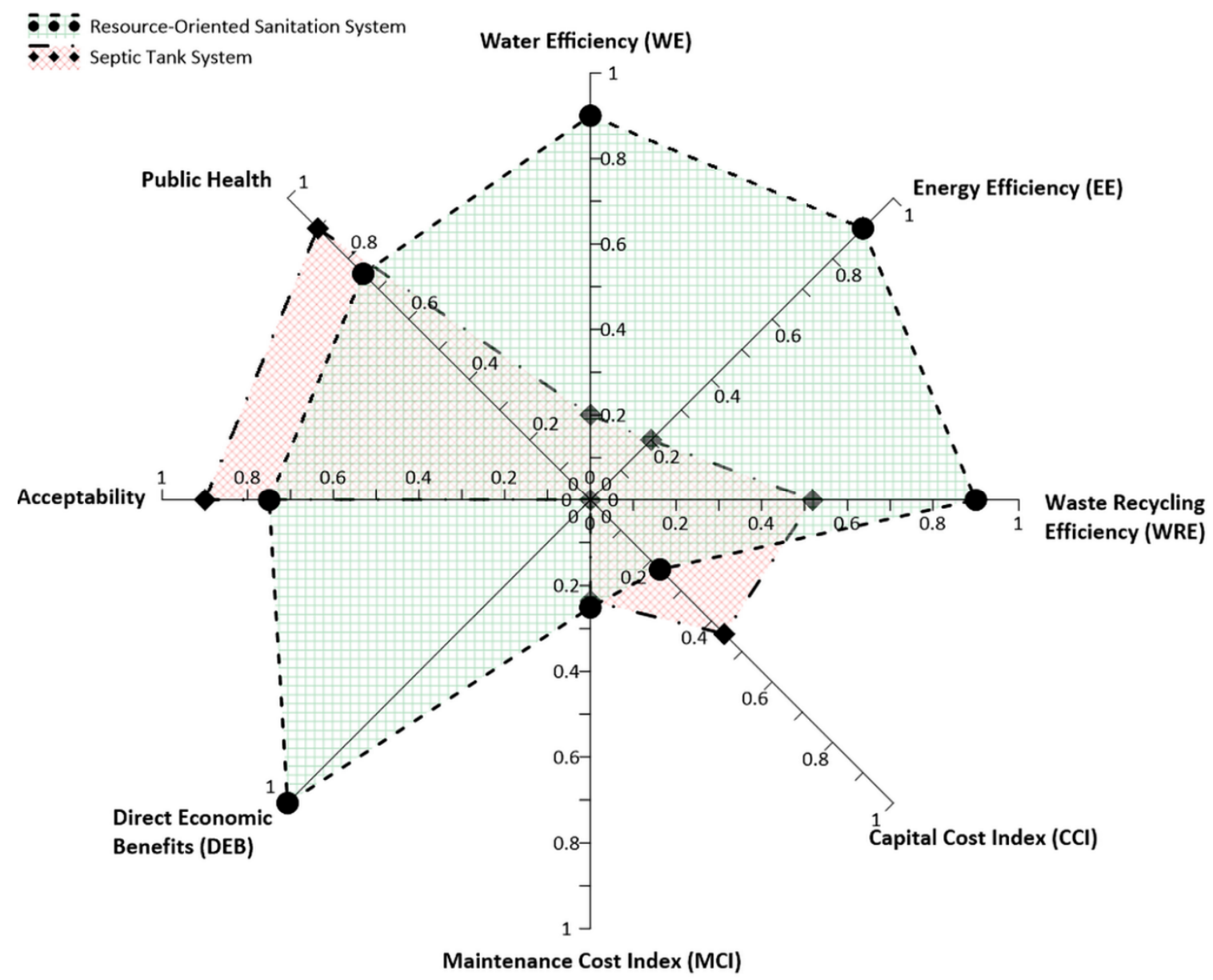

Figure 2. Comparison of sanitation sustainability index for septic tank and resource-oriented sanitation systems in South Korea.

The social sub-index of ROS systems is considered lower than that of a septic tank. Conducting in-depth studies on the social acceptability of sanitation systems in South Korea can provide a better assessment for this sub-index. Conducting safe, natural treatment methods for urine and feces, and providing sustainable operation monitoring, regular maintenance and training for users are beneficial actions for increasing the social sub-index scores of the ROS system.

\subsection{Sensitivity Analysis for SSI}

Figure 3 presents the sensitivity analysis for the studied sanitation systems. For the septic tank system, after social sub-indexes, the following descending order of variables in terms of their impact on SSI apply: $E_{C}, V_{C}, M_{E}, C_{E}$, and WRE. However, for the ROS system, DEBs and WRE have the greatest impact, while $\mathrm{CC}_{\mathrm{E}}$, acceptability, public health, and $\mathrm{MC}_{\mathrm{E}}$ indicators have notable impacts. The sensitivity of other variables was negligible. 
(a)

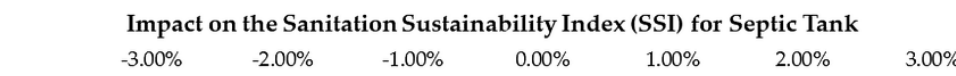

Energy Consumption to Treat Wastewater from a Single Use (EC)

Water Consumption for a Single Use (VC)

Estimated Maintenance Cost (MCE)

Estimated Capital Cost (CCE)

Waste Recycling Efficiency (WRE) $3.00 \%$

(b)
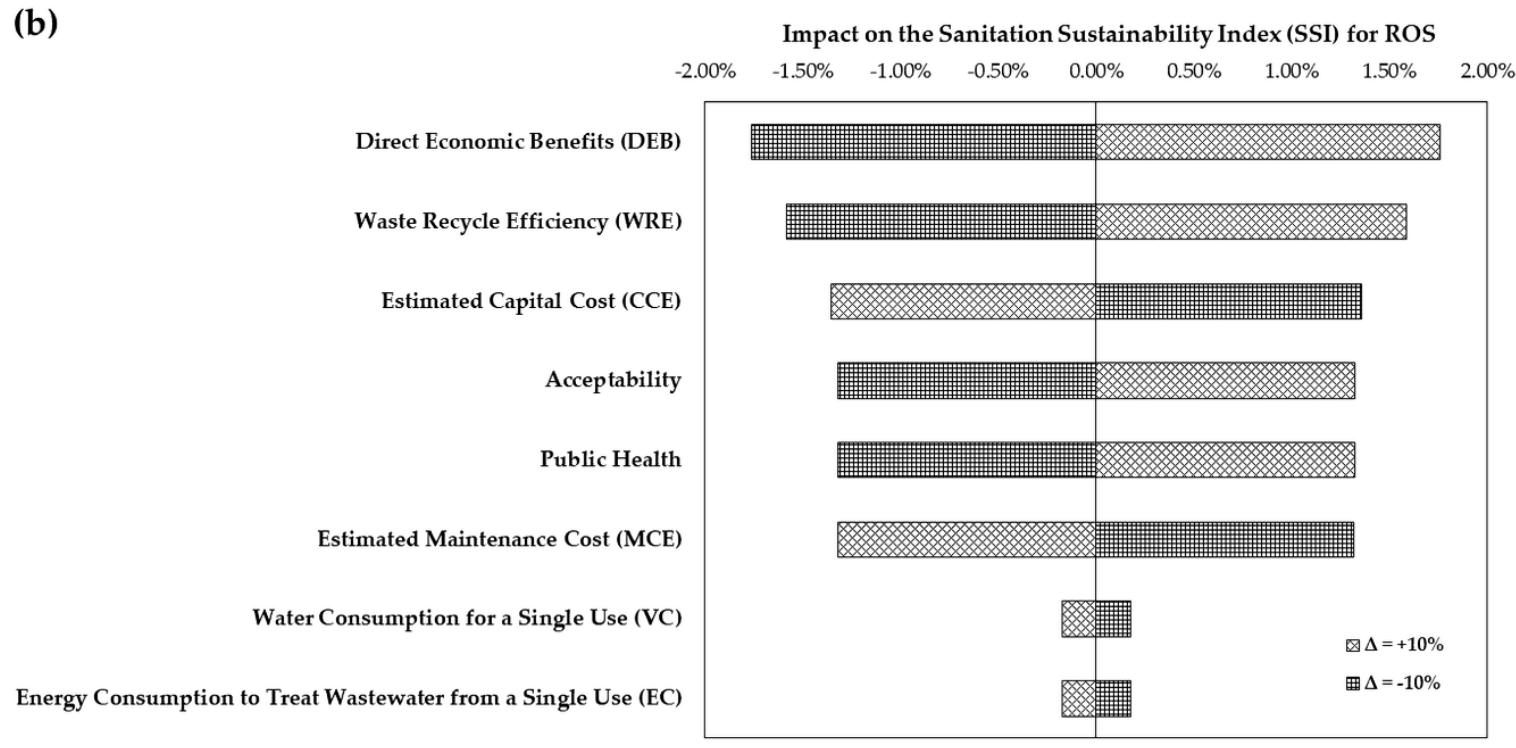

Figure 3. Sensitivity analysis for sanitation sustainability index calculated for (a) septic tank and (b) resource-oriented sanitation systems in South Korea.

These results indicate that for South Korea, which has a notably long history in recycling human waste as fertilizer, the development of an ROS system for waste recycling and utilization can potentially increase the SSI. Additionally, reducing the $\mathrm{CC}_{\mathrm{E}}$ and $\mathrm{MC}_{\mathrm{E}}$ by utilizing local labor and materials can also significantly increase the ROS system sustainability.

\subsection{Statistical Distribution Forecast for SSI}

Figure 4 presents the results of forecasting the SSI statistical distributions for both sanitation systems. Results for 1,000,000 trials yielded a mean of $0.36(95 \% \mathrm{CI}=0.24-0.50)$ and $0.67(95 \% \mathrm{CI}=$ $0.55-0.75)$ for septic tank and ROS systems, respectively. The calculated SSI scores for both systems (0.42 for septic tank system and 0.71 for ROS system) are within the $95 \% \mathrm{CI}$, and do not have a significant difference in the forecasted mean. Similar trends were observed for the forecasted statistical distributions of WE, EE, WRE, CCI, and MCI, and these results are presented as Supplementary Material. 
(a) Sanitation Sustainability Index (SSI) for Septic Tank in South Korea

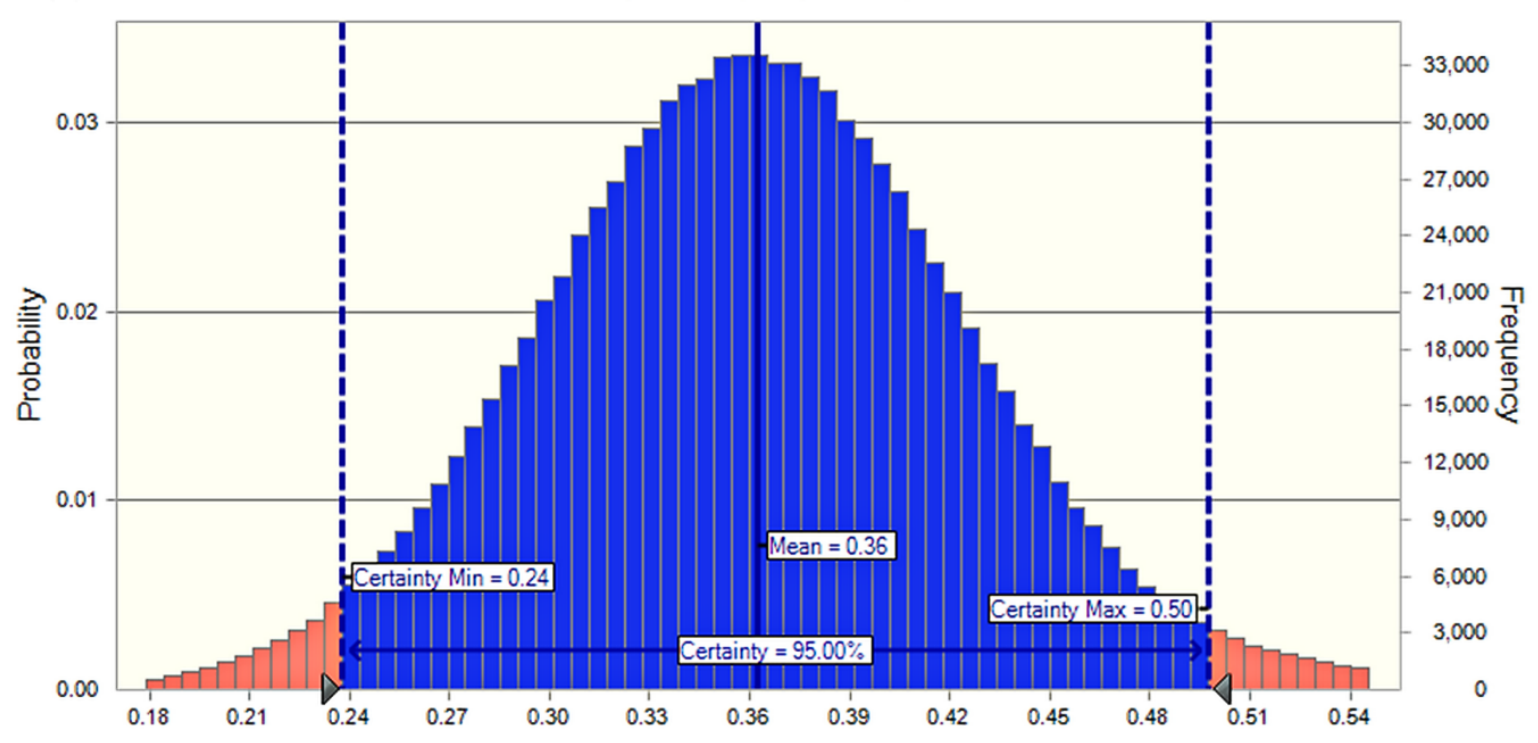

(b) Sanitation Sustainability Index (SSI) for Resource-Oriented Sanitation in South Korea

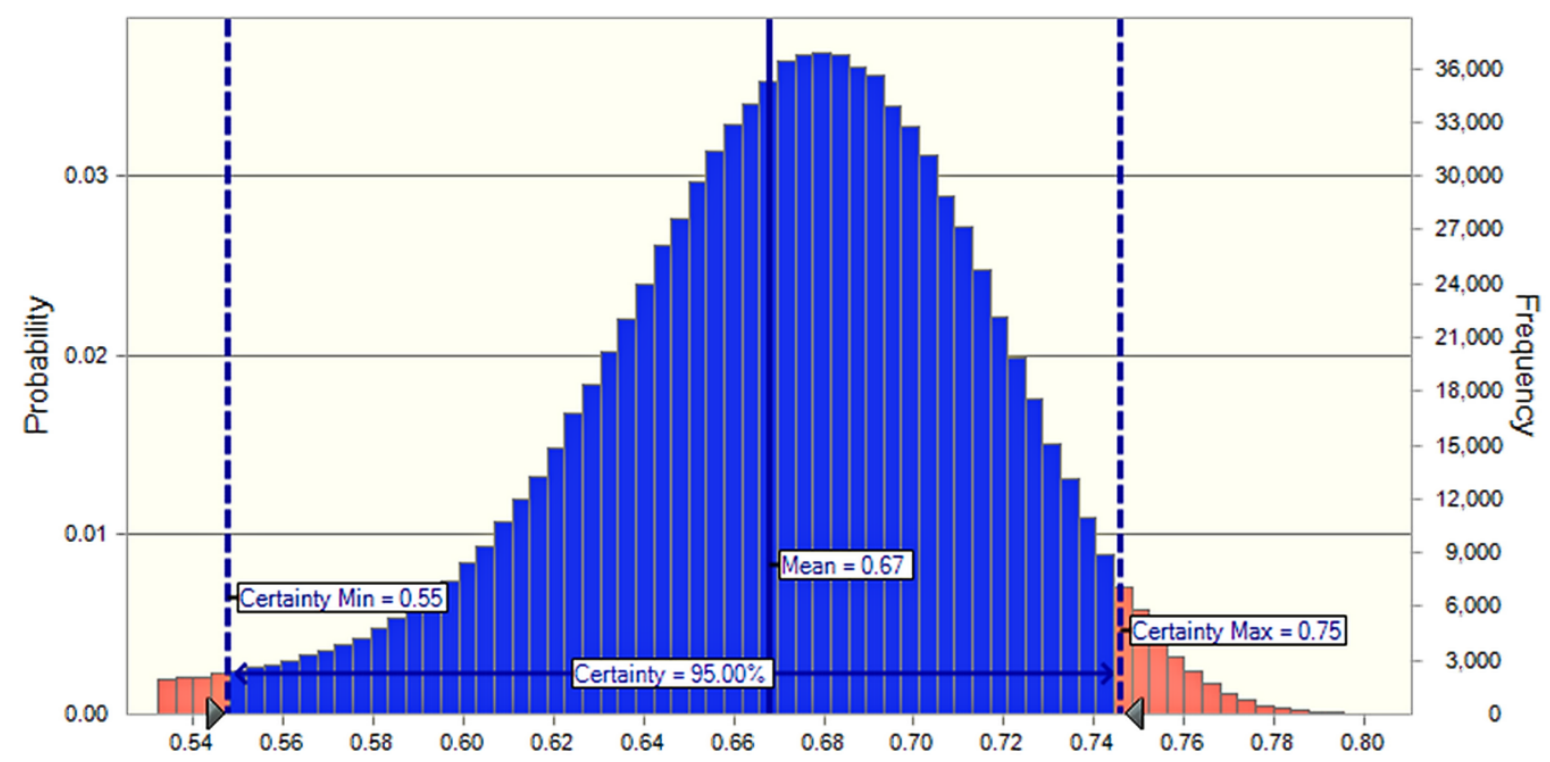

Figure 4. Forecasted statistical distribution of sanitation sustainability index calculated for (a) septic tank and (b) resource-oriented sanitation systems in South Korea.

The results concluded that the SSI could be a reliable index as a means for evaluating the sustainability of a sanitation system, as well as comparing the sustainability of different sanitation systems.

\section{Discussion}

The proposed SSI can be considered a tool to evaluate the sustainability of any local sanitation system and its associated infrastructure. It can be used to evaluate the sustainability of a single sanitation system, as well as to compare two or more systems. The index includes sub-indexes, which can provide technical, economic, and social evaluations. Overall, SSI can be a useful tool for sanitation system designers, managers, and developers. Meanwhile, decision-makers can also use SSI to compare different systems to choose the most sustainable one for implementation. 
The sub-indexes and their variables are based on the understanding of the sanitation status of the community, where the system is going to be implemented. Accordingly, the scope of the index is narrowed to one specific community. This means that the value of the index is specifically valid for a particular community and may differ between communities. This feature gives sufficient flexibility to the index, rendering it entirely replicable. Nevertheless, in case of availability of sufficient data reflecting the nation-wide sanitation status, SSI can also be used to evaluate sanitation systems at a national level.

The variables of all sub-indexes are also compatible with time. Community-based variables, such as $\mathrm{V}_{\mathrm{M}}, \mathrm{E}_{\mathrm{M}}, \mathrm{CC}_{\mathrm{M}}$, and $\mathrm{MC}_{\mathrm{M}}$, can be defined based on the predicted changes to the community (e.g., increase in population). Accordingly, the dimensionless index can also provide a vision about the sustainability of the assessed system in the future.

The flexibility and applicability of this index can be compared with that of the one suggested by Iribarnegaray et al., which also evaluates access to clean and safe water [36]. Most of the available indexes proposed to evaluate water and sanitation sustainability are useful in evaluating systems that are already implemented. Although all these indexes are defined with a similar objective, the SSI is used to evaluate the sustainability of sanitation systems before implementation, which can save time, money, and resources. Furthermore, its technical and economic sub-indexes are defined based on logical and simple equations, reflecting differences between the evaluated systems and the current norms of the community. Moreover, these sub-indexes can determine the potential of the system for waste recycling and reuse as well as provisions for economic benefits.

SSI applicability was demonstrated for evaluating the implementation of a septic tank and an ROS system in a suburban area of Seoul. Results indicated higher sustainability for the ROS system compared to the more commonly used septic tank system. Implementations of ROS systems are limited to the demonstration level through limited small projects.

A notable feature of the SSI is its use in determining the fate of wastes as well as the economic and social aspects of a sanitation system. Considering the high potential of an ROS system for waste recycling, it can be concluded that it is entirely sustainable in both technical and economic aspects. The index clearly shows that the implementation of the ROS has a higher capital cost, with the potential for providing direct economic benefits, which can cover both capital and maintenance costs.

With regard to social evaluation, the index assesses the acceptability and provision of public health services for a sanitation system. South Korea, along with some other Asian countries, has a historical background of using source-separated urine and feces as fertilizer [5]. Accordingly, ROS can be quite acceptable if more of them are implemented. Meanwhile, more efficient and hygienic maintenance and waste treatment processes can notably increase the social evaluation score of an ROS system. In addition, conducting a sensitivity analysis can determine the target parameters or sub-indexes of SSI for a specific sanitation system regarding a specific community. Accordingly, giving priority to these parameters might be useful to increase the sustainability of the sanitation system in the specific community.

The reliability of the SSI was examined through Monte Carlo simulation to forecast the statistical distribution of the calculated SSI scores. It became clear that the results were within the $95 \% \mathrm{CI}$. Accordingly, the index can be reliably applied to any other community with different settings when the only requirement for the SSI input is relevant local data.

\section{Limitations of SSI}

Although SSI is proposed to improve sanitation in communities worldwide, the approach described in this study may not be the ideal one.

For example, the economic sub-indexes are based on specific estimations that rely on the current financial status of the community. However, the economic situation of a community can be profoundly affected by unpredicted social events such as a pandemic. 
Moreover, the social sub-indexes are heavily dependent on the availability of related studies and databases. Especially for newly developed sanitation systems, there is a demand for previous pilot projects that investigated the acceptability and efficiency of the system in improving public health by reducing the risk of sanitation-related illnesses. The investigation methods depend on the community culture and available resources for the investigation. The role of SSI is to reflect and simplify the results of these investigations. Accordingly, to use SSI, prior local studies on economic and social perspectives of the community are crucial.

\section{Conclusions}

In this paper, the SSI is proposed as an index to evaluate the sustainability of sanitation systems intended to be implemented in a community. The SSI is simple to calculate, and its variables are flexible, time compatible, and entirely dependent on the characteristics of the community where the system will be implemented. It considers the technical, economic, and social aspects of the system. Therefore, the SSI is a reliable indicator to evaluate different sanitation systems, compare their sustainability, and choose the most suitable type for a specific community.

The applicability of the SSI has been demonstrated for South Korea by evaluating the implementation of a septic tank and an ROS system. A sensitivity analysis was performed to determine the variables that had significant impact. Statistical distribution was forecasted for the SSI of both systems and proved that the calculated SSI is within the $95 \%$ confidence interval.

The SSI can evaluate the sustainability of any sanitation system, considering the current water and sanitation condition of the community before implementation. This feature of SSI can make it useful for choosing the best sanitation system for a community, especially in remote and rural areas where access to clean water and sanitation is limited and inadequate.

The index applies to any community, and its prerequisite is relevant technical, economic, and social data. Accordingly, the provision of comprehensive, relevant databases for the community is essential. The lack of such databases causes limitations, especially for calculating the SSI social sub-index. Consequently, the development of a standard survey to determine social acceptability, cultural sanitation aspects, and public health indicators will be advantageous to further develop the SSI. Thus, studies on customizing the SSI and defining new variables or sub-indexes for specific communities will make this index more beneficial.

Supplementary Materials: The following are available online at http://www.mdpi.com/2071-1050/12/17/6937/s1: Sustainability Evaluation Results for Implementation of Septic Tank and Resource-Oriented Sanitation Systems in South Korea.

Funding: This research received no external funding.

Acknowledgments: The author's profound gratitude goes to Eng. Farid Hashemi, for his kind support and efforts. This article was written during the peak of the COVID-19 pandemic. The author would like to express his deepest sympathies to people worldwide who have faced losses due to this pandemic. Sanitation and hygiene have become even more critical now, and the author will continue to contribute toward achieving global sustainable and adequate sanitation, especially for those in remote and rural areas. The author wishes for the day where no one is left behind regarding access to clean water and sanitation and hopes that everyone stays safe and sound.

Conflicts of Interest: The author declares no conflict of interest.

\section{References}

1. UNICEF; WHO. Progress on Household Drinking Water, Sanitation and Hygiene 2000-2017: Special Focus on Inequalities; United Nations Children's Fund (UNICEF): New York, NY, USA; World Health Organization: New York, NY, USA, 2019; ISBN 978-92-806-5036-5.

2. UN Sustainable Development Goal 6. Available online: https://sustainabledevelopment.un.org/sdg6 (accessed on 24 March 2020).

3. Hyun, C.; Burt, Z.; Crider, Y.; Nelson, K.L.; Prasad, C.S.S.; Rayasam, S.D.G.; Tarpeh, W.; Ray, I. Sanitation for Low-Income Regions: A Cross-Disciplinary Review. Annu. Rev. Environ. Resour. 2019, 44, 287-318. [CrossRef] [PubMed] 
4. Jain, A.; Wagner, A.; Snell-Rood, C.; Ray, I. Understanding open defecation in the age of Swachh Bharat Abhiyan: Agency, accountability, and anger in rural Bihar. Int. J. Environ. Res. Public Health 2020, 17, 1384. [CrossRef]

5. Han, M.; Hashemi, S. Sanitation revolution: From waste to resource. Desalin. Water Treat. 2017, 91, 305-310. [CrossRef]

6. Langergraber, G.; Muellegger, E. Ecological Sanitation-A way to solve global sanitation problems? Environ. Int. 2005, 31, 433-444. [CrossRef] [PubMed]

7. Schwemlein, S.; Cronk, R.; Bartram, J. Indicators for monitoring water, sanitation, and hygiene: A systematic review of indicator selection methods. Int. J. Environ. Res. Public Health 2016, 13, 333. [CrossRef] [PubMed]

8. Cronk, R.; Luh, J.; Meier, B.M.; Bartram, J. The Water, Sanitation, and Hygiene Performance Index: A Comparison of Country Performance in Realizing Universal WaSH; University of North Carolina at Chapel Hill: Chapel Hill, NC, USA, 2015. [CrossRef]

9. UNDP. Human Development Index (HDI)|Human Development Reports. Available online: http://hdr.undp. org/en/content/human-development-index-hdi/ (accessed on 24 March 2020).

10. Lundin, M.; Molander, S.; Morrison, G.M. A set of indicators for the assessment of temporal variations in the sustainability of sanitary systems. Water Sci. Technol. 1999, 39, 235-242. [CrossRef]

11. Iribarnegaray, M.A.; Copa, F.R.; Gatto D’Andrea, M.L.; Arredondo, M.F.; Cabral, J.D.; Correa, J.J.; Liberal, V.I.; Seghezzo, L. A comprehensive index to assess the sustainability of water and sanitation management systems. J. Water Sanit. Hyg. Dev. 2012, 2, 205-222. [CrossRef]

12. Hashemi, S.; Boudaghpour, S. Economic Analysis and Probability of Benefit of Implementing Onsite Septic Tank and Resource-Oriented Sanitation Systems in Seoul, South Korea. Environ. Technol. Innov. 2020, 18, 100762. [CrossRef]

13. Simha, P.; Ganesapillai, M. Ecological Sanitation and nutrient recovery from human urine: How far have we come? A review. Sustain. Environ. Res. 2017, 27, 107-116. [CrossRef]

14. Hashemi, S.; Han, M.; Kim, T. Optimization of fertilization characteristics of urine by addition of Nitrosomonas europaea bio-seed. J. Sci. Food Agric. 2016, 96, 4416-4422. [CrossRef]

15. Hashemi, S.; Han, M. Optimizing source-separated feces degradation and fertility using nitrifying microorganisms. J. Environ. Manag. 2018, 206, 540-546. [CrossRef] [PubMed]

16. Hashemi, S.; Han, M.; Namkung, E. Fate of Fecal Indicators in Resource-Oriented Sanitation Systems Using Nitrifying Bio-Treatment. Int. J. Environ. Res. Public Health 2018, 15, 164. [CrossRef] [PubMed]

17. Simha, P.; Lalander, C.; Nordin, A.; Vinnerås, B. Alkaline dehydration of source-separated fresh human urine: Preliminary insights into using different dehydration temperature and media. Sci. Total Environ. 2020, 733, 139313. [CrossRef] [PubMed]

18. Katukiza, A.Y.; Ronteltap, M.; Oleja, A.; Niwagaba, C.B.; Kansiime, F.; Lens, P.N.L. Selection of sustainable sanitation technologies for urban slums-A case of Bwaise III in Kampala, Uganda. Sci. Total Environ. 2010, 409, 52-62. [CrossRef] [PubMed]

19. Tobias, R.; O’Keefe, M.; Künzle, R.; Gebauer, H.; Gründl, H.; Morgenroth, E.; Pronk, W.; Larsen, T.A. Early testing of new sanitation technology for urban slums: The case of the Blue Diversion Toilet. Sci. Total Environ. 2017, 576, 264-272. [CrossRef] [PubMed]

20. Hashemi, S.; Han, M. Field evaluation of the fertilizing potential of biologically treated sanitation products. Sci. Total Environ. 2019, 650, 1591-1598. [CrossRef]

21. Hashemi, S.; Han, M. Control of urine odor in different sanitation practices and its implication on water saving. J. Water Sanit. Hyg. Dev. 2017, 7, 156-162. [CrossRef]

22. Hashemi, S.; Han, M.; Kim, T. Identification of urine scale problems in urinals and the solution using rainwater. J. Water Sanit. Hyg. Dev. 2015, 5, 322-329. [CrossRef]

23. Hashemi, S.; Han, M.; Kim, T. The effect of material and flushing water type on urine scale formation. Water Sci. Technol. 2015, 72, 2027-2033. [CrossRef]

24. Koottatep, T.; Pussayanavin, T.; Polprasert, C. Nouveau design solar septic tank: Reinvented toilet technology for sanitation 4.0. Environ. Technol. Innov. 2020, 19, 100933. [CrossRef]

25. Flanagan, C.P.; Randall, D.G. Development of a novel nutrient recovery urinal for on-site fertilizer production. J. Environ. Chem. Eng. 2018, 6, 6344-6350. [CrossRef] 
26. Tahulela, A.C.; Ballard, H.H. Developing the Circular Economy in South Africa: Challenges and Opportunities. In Sustainable Waste Management: Policies and Case Studies; Ghosh, S., Ed.; Springer: Singapore, 2020; pp. 125-133. [CrossRef]

27. Simha, P.; Lalander, C.; Vinnerås, B.; Ganesapillai, M. Farmer attitudes and perceptions to the re-use of fertiliser products from resource-oriented sanitation systems-The case of Vellore, South India. Sci. Total Environ. 2017, 581-582, 885-896. [CrossRef] [PubMed]

28. Drangert, J.O.; Nawab, B. A cultural-spatial analysis of excreting, recirculation of human excreta and health-The case of North West Frontier Province, Pakistan. Health Place 2011, 17, 57-66. [CrossRef] [PubMed]

29. Mariwah, S.; Drangert, J.O. Community perceptions of human excreta as fertilizer in peri-urban agriculture in Ghana. Waste Manag. Res. 2011, 29, 815-822. [CrossRef] [PubMed]

30. Hashemi, S.; Han, M. Methods for controlling stored urine odor in resource-oriented sanitation. J. Water Sanit. Hyg. Dev. 2017, 7, 507-514. [CrossRef]

31. Mkhize, N.; Taylor, M.; Udert, K.M.; Gounden, T.G.; Buckley, C.A. Urine diversion dry toilets in eThekwini municipality, South Africa: Acceptance, use and maintenance through users' eyes. J. Water Sanit. Hyg. Dev. 2017, 7, 111-120. [CrossRef]

32. Ssemugabo, C.; Halage, A.A.; Namata, C.; Musoke, D.; Ssempebwa, J.C. A socio-ecological perspective of the facilitators and barriers to uptake of water, sanitation and hygiene interventions in a slum setting in Kampala, Uganda: A qualitative study. J. Water Sanit. Hyg. Dev. 2020, 10, 227-237. [CrossRef]

33. Hashemi, S.; Han, M.; Kim, T.; Kim, Y. Innovative Toilet Technologies for Smart and Green Cities. In True Smart $\mathcal{E}$ Green Urban Technologies and Infrastructure Systems, Proceedings of the 8th Conference of the International Forum on Urbanism (IFoU), Incheon, Korea, 22-24 June 2015; Kim, D., Kim, S., Schuetze, T., Sohn, S., Chelleri, L., Ostermeyer, Y., Tieben, H., Wolfram, M., Eds.; International Forum on Urbanism (IFoU): Delft, The Netherlands, 2015; p. E013. [CrossRef]

34. Guerrini, A.; Romano, G.; Indipendenza, A. Energy efficiency drivers in wastewater treatment plants: A double bootstrap DEA analysis. Sustainability 2017, 9, 1126. [CrossRef]

35. MOE. Modernization of the Sewerage System in Korea; The International Bench Marking Network (IBNET): Washington, DC, USA, 2015. Available online: https://www.ib-net.org/docs/History_of_Korea|T1〉 textquoterights_sewerage_system_development.pdf (accessed on 24 March 2020).

36. Iribarnegaray, M.A.; Gatto D’Andrea, M.L.; Rodriguez-Alvarez, M.S.; Hernández, M.E.; Brannstrom, C.; Seghezzo, L. From indicators to policies: Open sustainability assessment in the water and sanitation sector. Sustainability 2015, 7, 14537-14557. [CrossRef] 